Article

\title{
Customized In Situ Functionalization of Nanodiamonds with Nanoparticles for Composite Carbon-Paste Electrodes
}

\author{
Raquel Montes ${ }^{1}$, Gerard Sánchez ${ }^{2}$, Jingjing Zhao ${ }^{3}$, Cristina Palet ${ }^{3}{ }^{1}$, Mireia Baeza ${ }^{2, *}$ and \\ Julio Bastos-Arrieta $4, *$ (D)
}

1 GENOCOV Research Group, Department of Chemical, Biological and Environmental Engineering, School of Engineering, Universitat Autònoma de Barcelona, Carrer de les Sitges, 08193 Bellaterra (Cerdanyola del Vallès), Spain; raquel.montes@uab.cat

2 GENOCOV Research Group, Department of Chemistry, Faculty of Science, Edifici C-Nord, Universitat Autònoma de Barcelona, Carrer dels Til·lers, 08193 Bellaterra (Cerdanyola del Vallès), Spain; gerarg_93@hotmail.com

3 Grup de Tècniques de Separació en Química, Department of Chemistry, Facultat de Ciències, Universitat Autònoma de Barcelona, Carrer dels Til-lers, 08193 Bellaterra (Cerdanyola del Vallès), Spain; zhaojingjing186@sina.com (J.Z.); cristina.palet@uab.cat (C.P.)

4 Physical Chemistry TU Dresden, Zellescher Weg 19, 01069 Dresden, Germany

* Correspondence: mariadelmar.baeza@uab.cat (M.B.); julio.bastos@tu-dresden.de (J.B.-A.)

Received: 22 May 2020; Accepted: 15 June 2020; Published: 17 June 2020

\begin{abstract}
The incorporation of nanomaterials on (bio)sensors based on composite materials has led to important advances in the analytical chemistry field due to the extraordinary properties that these materials offer. Nanodiamonds (NDs) are a novel type of material that has raised much attention, as they have the possibility of being produced on a large scale by relatively inexpensive synthetic methodologies. Moreover, NDs can present some other interesting features, such as fluorescence, due to surface functionalization and proved biocompatibility, which makes them suitable for biomedical applications. In addition, NDs can be customized with metallic nanoparticles (NPs), such as silver or gold, in order to combine the features of both. Raw NDs were used as modifiers of sensors due to the electrocatalytic effect of the $\mathrm{sp}^{2}$ and oxygenated species present on their surface. The aim of this research work is evaluating the applicability of NDs modified with silver (Ag@NDs) and gold (Au@NDs) nanoparticles for the development of a suitable (bio)sensing platform. A complete morphological and electrochemical characterization as a function of the prepared nanocomposite composition was performed in order to improve the electroanalytical properties of the developed (bio)sensors. In the present work, the optimal composition for Au@NDs present on the nanocomposite matrix is 3.5\% and the one for Ag@NDs is 1\%. Good results were obtained in the evaluation of the optimal composition towards hydrogen peroxide and glucose as a model analyte using a (bio)sensor based on graphite-epoxy-Ag@NDs (17:82:1).
\end{abstract}

Keywords: carbon nanostructures; nanodiamonds; graphite; optimal composition; metal nanoparticles; electrochemical (bio)sensor; surface functionalization

\section{Introduction}

Carbon-based nanocomposite materials used for (bio)sensing platforms, include different carbon nanallotropic forms (e.g., graphite, graphene) that led to electroanalytical improvements [1,2] in comparison to conventional solid electrodes, such as robustness, renewable surface, or a small background current. Composite electrodes can be customized with a variety of nanomaterials 
including electrocatalysts, enzymes, metal nanoparticles (NPs), quantum dots, proteins, ligands, and other chemical (bio)recognition agents [3,4]. In addition, this modification can be either within the matrix itself or on the surface.

Nanodiamonds (NDs) are another carbon nanoallotrope that recently have been widely introduced as components of (bio)sensing platforms. This is due to their cost effective large scale production with narrow size distribution, biocompatibility, and their surface chemistry, that make them suitable for further customization with different functional moieties [5]. Even though bulk diamonds are well known isolators, features attributed to the nanoscale make NDs to be electrochemically active $[6,7]$. This can be explained because of the existence of discrete electronic states within the NDs band gap (overlapping of surface $\mathrm{sp}^{2}$ orbitals), which provides NDs with a surface suitable to undergo redox reactions, and consequently have an electrochemical response $[8,9]$.

All these characteristics make it interesting to evaluate the effect of the customization of NDs to carbon nanocomposite (bio)sensors by three different routes [10]:

(a) Covalent or noncovalent functionalization of the NDs with the modifiers and their subsequent mixing within the polymer.

(b) Directly introduce the modifiers in the carbon/polymer matrix during the preparation, which remain embedded or trapped in the nanocomposite.

(c) Immobilization of the (bio)species on the surface of the already prepared nanocomposite electrode [11].

The final properties of the nanocomposite electrodes depend on their composition, which influence highly on their electroanalytical response [12]. Up to now the principle applied to the optimization of the composite proportions has been done using the percolation theory, under the criteria of maximizing the conductive particle loading, without losing its physical and mechanical stability. The nanocomposite becomes conductive above a critical value, which is called the percolation threshold and defines the insulator-conductor transition. At this point, the first conductive network is formed through the matrix. In order to reach the electrical percolation threshold and therefore be electrically conductive, a direct physical connection or overlapping of the conductive filler is not necessary [13]. Recently, complementary strategies of characterization have been established, which demonstrate that if the composite proportions are optimized, the response of the electrode will be improved [11,13].

Electrochemical impedance spectroscopy (EIS) is a powerful technique, which offers information about the electron-transfer rate $\left(R_{c t}\right)$, the double-layer capacitance $\left(C_{d l}\right)$, contact resistance and resistance of the solution $\left(R_{\Omega}\right)$ [14]. The electroanalytical properties required by an electrode in order to ensure enough sensitivity, a high signal-to-noise ratio, and low detection limit are: High electron-transfer rate, the lowest ohmic resistance, and double-layer capacitance. Using the EIS technique, it is possible to determine the optimal composite composition that exhibits these electrochemical properties associated with the improvement of the electroanalytical performance. Complementary techniques such as cyclic voltammetry (CV) allows obtaining supplementary information obtained by the EIS [15].

Montes et al. (2015) studied the optimization of the conductive particle distribution and the amount of the biological material inside the nanocomposite electrodes. The authors showed how these factors led to an improvement of electrochemical properties such as signal stability and limit of detection. In this case, for biocomposites based on graphite-epoxy and modified with glucose oxidase (GOD), as an enzyme model [16]. In addition, this work demonstrated that optimized electrodes with $17 \%$ of graphite and $1 \%$ of biological material presented good sensitivity and wide linear range towards glucose, as well as the limit of detection achieved was lower compared to the nonoptimized biocomposites. Later, Montes et al. (2016) reported that optimized immunosensors based on $17 \%$ of graphite and epoxy and $0.9 \%$ of RIgG on the matrix allowed obtaining the lower limit of detection using a competitive assay [17].

X-ray photoelectron spectroscopy (XPS) studies suggest that NDs are mainly composed of nonconductive $\mathrm{sp}^{3}$ carbon (resulting in low electroconductivity). Despite this, incorporating NDs 
to sensing platforms produces an electrocatalytic effect regarding changes in the mass transport. This variation can be explained because NDs block the underlying electroactive graphite matrix, creating a random array of graphite microelectrodes [9]. These facts encourage analyzing the combined effect of incorporating NDs functionalized with conductive metal NPs.

Consequently, $17 \%$ of graphite was fixed as a conductive material and the effect of the different proportions of NDs functionalized with silver and gold nanoparticles has been evaluated. Moreover, these studies concluded that to obtain a low limit of detection the amount of graphite loading it is more critical than the biological material loading in the biocomposites.

We report here the construction and characterization of nanocomposites based on graphite-epoxy and modified nanodiamonds functionalized with metallic NPs (Ag@NDs and Au@NDs). The electrochemical properties of such electrodes were studied in order to optimize the electrochemical behavior. The electroanalytical response of the optimized nanocomposites was evaluated using one reference analyte such as hydrogen peroxide. Finally, as a proof of concept of a suitable (bio)sensing platform, the optimized nanocomposite electrode was modified with glucose oxidase and applied to the detection of its enzymatic substrate.

\section{Materials and Methods}

\subsection{Apparatus}

EIS and voltammetric measurements (CV) were performed using a computer controlled Autolab PGSTAT12 potentiostat/galvanostat (EcoChemie, Utrech, The Netherlands) with a three-electrode configuration. A platinum-based electrode 53-671 (Crison Instruments, Alella, Barcelona, Spain), an $\mathrm{AgCl}$ covered silver wire, and the constructed graphite nanocomposite electrodes were used as a counter, reference, and working electrodes, respectively.

Linear-sweep voltagrams were performed using a computer controlled Autolab PGSTAT12 potentiostat/galvanostat (EcoChemie, Utrech, The Netherlands) with a three-electrode configuration. A single junction reference electrode $\mathrm{Ag} / \mathrm{AgCl}$ Orion 900,100 (Thermo Electron Corporation, Beverly, MA, USA) with $3.0 \mathrm{M} \mathrm{KCl}$ as internal reference solution and platinum-based electrode were used as reference and auxiliary, respectively, and graphite nanocomposite electrodes as working electrodes.

Amperometric measurements were done using an amperemeter LC-4C (Bioanalytical Systems Inc., West Lafayette, IN, USA). Electroanalytical experiments were carried out using a three-electrode configuration. A single junction reference electrode $\mathrm{Ag} / \mathrm{AgCl}$ Orion 900,100 (Thermo Electron Corporation, Beverly, MA, USA) with $3.0 \mathrm{M} \mathrm{KCl}$ as internal reference solution and platinum-based electrode were used as reference and auxiliary, respectively. The graphite nanocomposites electrodes were used as a working electrode. A magnetic stirrer at constant rpm provided the convective transport during the amperometric measurements.

Transmission electron microscopy (TEM) images were obtained by the high-resolution transmission electron microscopy (HR-TEM) technique using the JEOL JEM-1400 unit (Tokyo, Japan) with an acceleration voltage of $120 \mathrm{kV}$. At the same time, the energy dispersive spectroscopy (EDS) analysis was used for the qualitative determination of NPs presence.

Scanning electron microscopy (SEM) images were obtained with the Zeiss EVO ${ }^{\circledR}$ MA 10 unit (Jenna, Germany) with an acceleration voltage of $15 \mathrm{kV}$.

\subsection{Chemical Reagents}

Graphite powder (average particle size of $50 \mu \mathrm{m}$ ) was received from Merck Millipore (Darmstadt, Germany) and the epoxy resin EpoTek H77 with the corresponding hardener was supplied by Epoxy Technologies (Billerica, MA, USA). Nanodiamonds ( $>87 \%$ ) were obtained from PlasmaChem (Berlin, Germany). Potassium chloride $(99.5 \%)$, potassium ferrocyanide trihydrate $(>99 \%)$, potassium ferricyanide (III) $(99 \%)$, nitric acid $(99.5 \%)$, potassium nitrate $(99 \%)$, potassium hydrogenphosphate $(99.5 \%)$, potassium dihidrogenpohsphate $(99.5 \%)$, silver nitrate $(\geq 99 \%)$, gold chloride trihydrate 
( $\geq 99.9 \%)$, sodium chloride $(99.5 \%)$, sodium borohydride, chloroauric acid $(\leq 99.9 \%)$, hydrogen peroxide (30\%), glucose oxidase VII from Aspergillus niger (174,400 units/g), D-(+)-Glucose ( $\geq 99.5 \%)$, and bovine serum albumin, all of them were supplied by Sigma-Aldrich (St. Louis, MO, USA). Phosphate buffers were prepared from the potassium hydrogenphosphate $\left(\mathrm{K}_{2} \mathrm{HPO}_{4}\right)$ and dihydrogenphosphate $\left(\mathrm{KH}_{2} \mathrm{PO}_{4}\right)$ salts in Milli-Q water (Millipore, Billerica, MA, USA). All the dissolutions were prepared using deionized water from the Milli-Q system (Millipore, Billerica, MA, USA) with a resistivity value of $18.2 \mathrm{M} \Omega \cdot \mathrm{cm}$.

\subsection{Synthesis of $A g @ N D s$ and $A u @ N D s$}

Functionalization of NDs with silver (Ag@NDs) and gold nanoparticles (Au@NDs) was carried out following an Intermatrix Synthesis approach reported in previous works [18,19], which is based on ion exchange and reduction stages to obtain a favorable distribution of nanoparticles. The NDs surface was activated with carboxylic groups by dispersing them in a $2.5 \mathrm{M}$ nitric acid and placing them all in an ultrasound bath for $2 \mathrm{~h}$. These groups were converted to the $\mathrm{Na}^{+}$form by treating them with a $1.0 \mathrm{M} \mathrm{NaCl}$ solution with mechanical stirring for $2 \mathrm{~h}$ (see Equation (1)).

$$
\left[\mathrm{NDs}-\mathrm{COO}^{-} \mathrm{H}^{+}\right]+\mathrm{Na}^{+} \rightarrow\left[\mathrm{NDs}-\mathrm{COO}^{-} \mathrm{Na}^{+}\right]+\mathrm{H}^{+}
$$

Firstly, the Ag-NPs precursor $\left(\mathrm{Ag}^{+}\right)$is fixed on the NDs by an ion exchange stage with $\mathrm{AgNO}_{3}$, following Equation (2):

$$
\left[\mathrm{NDs}-\mathrm{COO}^{-} \mathrm{Na}^{+}\right]+\mathrm{Ag}^{+} \rightarrow\left[\mathrm{NDs}-\mathrm{COO}^{-} \mathrm{Ag}^{+}\right]+\mathrm{Na}^{+}
$$

Then, the appearance of the $\mathrm{Ag}^{0}-\mathrm{NPs}$ is achieved after the addition of $\mathrm{NaBH}_{4}$ (as the reducing agent). This reaction, leads the NDs to their $\mathrm{Na}^{+}$form again, making suitable further synthesis of $\mathrm{Ag}^{0}$-NPs by this approach (as can be seen in Equation (3)).

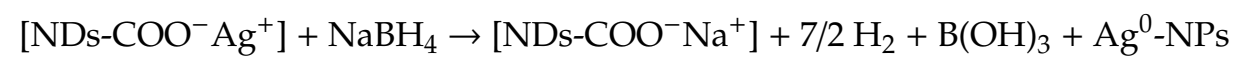

For the preparation of Au@NDs, a galvanic replacement strategy is needed, as the commonly used precursor of the $\mathrm{Au}^{0}$-NPs is negatively charged $\left(\mathrm{AuCl}_{4}{ }^{-}\right)$and therefore, its fixation on the carboxylic moieties is not possible. Thus, the difference of redox potentials between $\mathrm{Au}$ and $\mathrm{Ag}$ can be exploited, leading to a highly spontaneous galvanic replacement process to obtain $\mathrm{Au}^{0}$-NPs using the previously prepared $\mathrm{Ag}^{0}-\mathrm{NPs}$ as sacrificial templates (Equation (4)) [20].

$$
\mathrm{Ag}^{0}-\mathrm{NPs}+\mathrm{AuCl}_{4}^{-} \rightarrow \mathrm{Au}^{0}-\mathrm{NPs}+4 \mathrm{Cl}^{-}+3 \mathrm{Ag}^{+}
$$

\subsection{Fabrication of the Electrodes}

The preparation of the nanocomposite material was handmade by mixing the polymer Epotek H77A and its corresponding H77B hardener in a 20:3 $(w / w)$ ratio and adding the conducting filler nanomaterial, graphite. The composition was fixed at $17 \%$ of graphite loading as an optimal composition for the development of amperometric nanocomposites based on previous studies [16,17]. The composite was homogenized for $30 \mathrm{~min}$. After the homogenization time, the nanomaterial was incorporated, either raw NDs $(1 \%(w / w))$, NDs functionalized with gold nanoparticles $(1 \%, 2 \%$, and $3.5 \%(w / w))$, or NDs functionalized with silver nanoparticles $(1 \%, 2 \%$, and $3 \%(w / w))$. The composite paste was homogenized for an additional $15 \mathrm{~min}$ to assure the integration of the nanomaterial. The final nanocomposite paste electrode was allowed to harden during $24 \mathrm{~h}$ at $60^{\circ} \mathrm{C}$. Finally, the electrode surface was polished with different sandpapers of decreasing grain size. The final electrode dimensions were $28 \mathrm{~mm}^{2}$ and $3 \mathrm{~mm}$ for its geometric area and thickness, respectively. In Table 1, the different compositions evaluated on the present work are summarized. 
Table 1. Composition summary of fabricated nanocomposite electrodes.

\begin{tabular}{ccc}
\hline$\%$ Graphite/\% Epoxy $(w / w)$ & $\%$ Modifier $(w / w)$ & Nomenclature \\
\hline $17 \% / 83 \%$ & - & Bare electrode \\
$17 \% / 82 \%$ & $1 \%$ raw NDs & NDs electrode \\
$17 \% / 82 \%, 81 \%$, and $79.5 \%$ & $1 \%, 2 \%$, and 3.5\% Au@NDs & Au@NDs electrode \\
$17 \% / 82 \%, 81 \%$, and $80 \%$ & $1 \%, 2 \%$, and $3 \%$ Ag@NDs & Ag@NDs electrode \\
\hline
\end{tabular}

\subsection{Electrochemical Procedure}

EIS and CV measurements were carried out in a $0.1 \mathrm{M}$ potassium chloride solution containing $0.01 \mathrm{M}$ potassium ferricyanide/ferrocyanide under quiescent conditions. The optimal polarization potential of the studied analytes for each prepared nanocomposite electrode was obtained by linear scan voltammetry. This technique consists of registering the intensity while a potential sweep is done in a determined direction at a constant scan rate. The sweeps are carried out without force. For each of the studied analyte a sweep potential between 0 and $1.5 \mathrm{~V}$ was applied at a scan rate of $10 \mathrm{mV} \cdot \mathrm{s}^{-1}$. Firstly, a potential sweep is done on a $20 \mathrm{~mL}$ solution containing the support electrolyte, PBS at $\mathrm{pH}$ 7.0, and then successive sweeps are carried out on the same electrolytic solution after adding, in a consecutive way, different microvolumes of a solution containing the analyte of interest. For hydrogen peroxide measurements, consecutive microvolumes of a solution of $1 \mathrm{M}$ hydrogen peroxide solution were added until a final concentration of $27 \mathrm{mM} \mathrm{H}_{2} \mathrm{O}_{2}$ in the electrolytic media. For glucose measurements, consecutive microvolumes of a $1 \mathrm{M}$ glucose solution were added until a final concentration of $100 \mathrm{mM}$ glucose in the electrolytic media.

Amperometric detection of the different reference analytes (hydrogen peroxide and glucose) was made under force convection by stirring the electrolyte solution with a magnetic stirrer. The limit of detection (LOD) was estimated by the $S / N=3$ criterion [21]. The LOD was calculated three times $(n=3)$ and presented with their respective standard deviation.

\subsection{Surface Modification of the Nanocomposite Electrodes with Glucose Oxidase}

For the surface modification of the electrodes with glucose oxidase by the direct contact method, a stock solution is prepared from $15 \mathrm{mg}$ of GOD and $300 \mu \mathrm{L}$ of the bovine serum albumin (BSA) solution at $1 \%(p / v)$ in PBS. The electrode was incubated with the GOD solution using a thermomixer (Thermomixer comfort, Eppendorf AG, Hamburg, Germany) at $600 \mathrm{rpm}$ and $20^{\circ} \mathrm{C}$. Three different contact times were evaluated: $30 \mathrm{~min}, 1 \mathrm{~h}$ and $24 \mathrm{~h}$.

\section{Results}

\subsection{Characterization of the Modified NDs Containing Composites}

The characterization of the NDs before and after the process of modification with the metal NPs was screened by HR-TEM. Figure 1 shows the schematic representation of the NDs modification, together with the TEM images and the corresponding energy-dispersive X-ray spectroscopy (EDS) for all systems, NDs, Ag@NDs, and Au@NDs. A good monodispersity in the NDs size can be observed. Moreover, the average diameter of the NDs was $<4 \mathrm{~nm}(3.5 \pm 0.3)$, and when adding the silver and gold nanoparticles it increased up to $4.5 \pm 0.3 \mathrm{~nm}$ for the Ag@NDs and $6.7 \pm 0.5 \mathrm{~nm}$ for the Au@NDs. Furthermore, the EDS carried out for both Ag@NDs and Au@NDs systems confirmed the presence of the silver and gold NPs attached to the NDs. 
A)

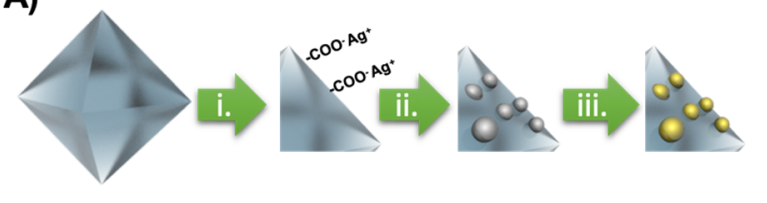

i. Loading of $\mathrm{Ag}$ ions (NP precursor)

ii. Redox Stage: synthesis of Ag-NPs

iii. Galvanic Replacement: Au-NPs

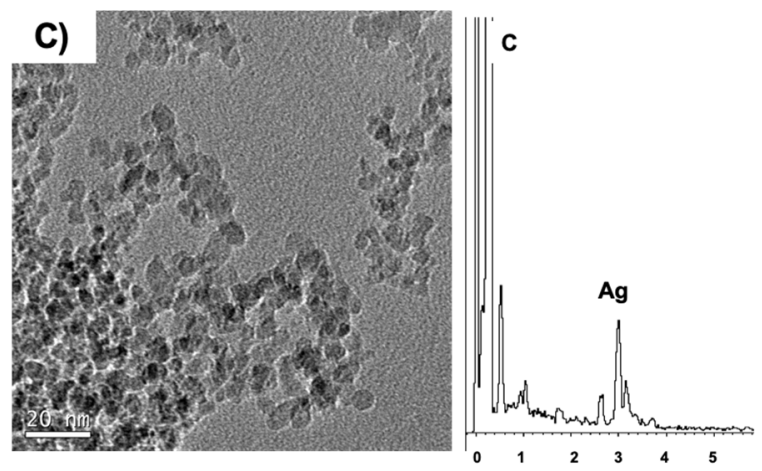

B)
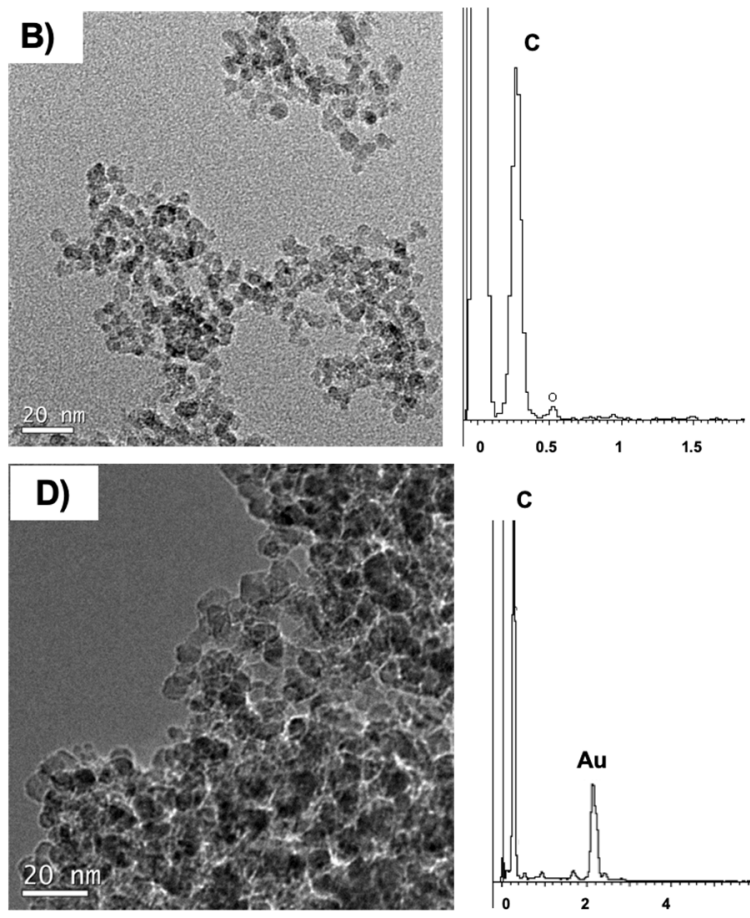

C

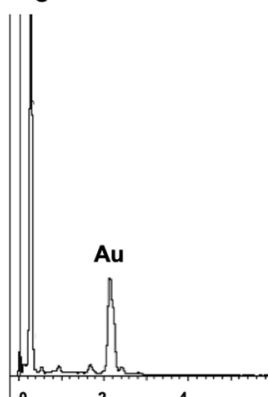

Figure 1. Scheme of the functionalization process (A), nanodiamonds (NDs) TEM image and EDS (B), silver nanodiamonds (Ag@NDs) TEM image and EDS (C), and gold nanodiamonds (Au@NDs) TEM image and EDS (D).

\subsection{Morphological Characterization by SEM}

The SEM images allowed observing qualitatively the roughness and the porosity of the nanocomposites surface constructed. Figure S1 shows SEM images obtained for each ND containing nanocomposite, in comparison to the bare electrode based on graphite-epoxy (Figure S1A) at different magnification levels. It can be seen that the incorporation of the different nanomaterials to the matrix at different amounts of gold and silver NPs: NDs (Figure S1B), Au@NDs (Figure S1C-E), and Ag@NDs (Figure S1F-H) does not modify the morphology and that in all cases, the surface presents a high homogeneity and certain roughness. This homogeneity assures better reproducibility during the analytical application.

\subsection{Impedimetric Characterization}

By means of the EIS technique, the electrochemical parameters of the different nanocomposite electrodes developed have been evaluated. The obtained values for $R_{\Omega}, R_{c t}$, and $C_{d l}$ allow predicting the electrochemical behavior as a function of the amount of nanomaterial (raw NDs, Au@NDs, or Ag@NDs) incorporated to the matrix. These properties have been compared to the ones of the bare electrode. The electroanalytical properties required by an electrode are the high electron-transfer rate (lower $R_{c t}$ ) and the lowest resistance of the solution $\left(R_{\Omega}\right)$ together with the lowest double-layer capacitance $\left(C_{d l}\right)$, in order to ensure high sensitivity and high signal-to-noise ratio and, therefore, low detection limits. The results (Figure S2A,B, Supplementary Materials) were obtained by fitting the impedance spectra to an equivalent Randles circuit (see Figure S2C, Supplementary Materials). This circuit was sufficiently suitable in order to adjust and obtain the values of $R_{\Omega}, R_{c t}$, and $C_{d l}$, from the point of view of the interfacial phenomena that takes places in the electrochemical cell [22]. Results for Au@NDs or $\mathrm{Ag} @ \mathrm{NDs}$ are presented separately.

Figure 2 presents the values for $R_{\Omega}, R_{c t}$, and $C_{d l}$ and shows the comparison between the bare electrode containing $17 \%$ graphite and the modified electrodes with $17 \%$ graphite plus $1 \%$ raw NDs and $17 \%$ graphite plus 1\%, $2 \%$, and 3.5\% Au@NDs. An increase in the electrochemical parameters' 
values are observed when $1 \%$ of raw NDs are incorporated to the matrix. This increase is associated to the separation in the conductive particles present on the surface electrodes. When it is related to the electrodes with different amounts of Au@NDs, the electrode with 17\% graphite plus 3.5\% Au@NDs is the one with the lowest ohmic resistance (238.64 $\Omega$ ) and charge-transfer resistance (513.54 $\Omega$ ). Regarding the double-layer capacitance values, no significant difference between the three electrode compositions containing gold is observed. According to these results, the one containing 3.5\% Au@NDs would be the optimal in this case due to this composition that presents the lowest ohmic resistance, charge-transfer resistance, and double-layer capacitance.

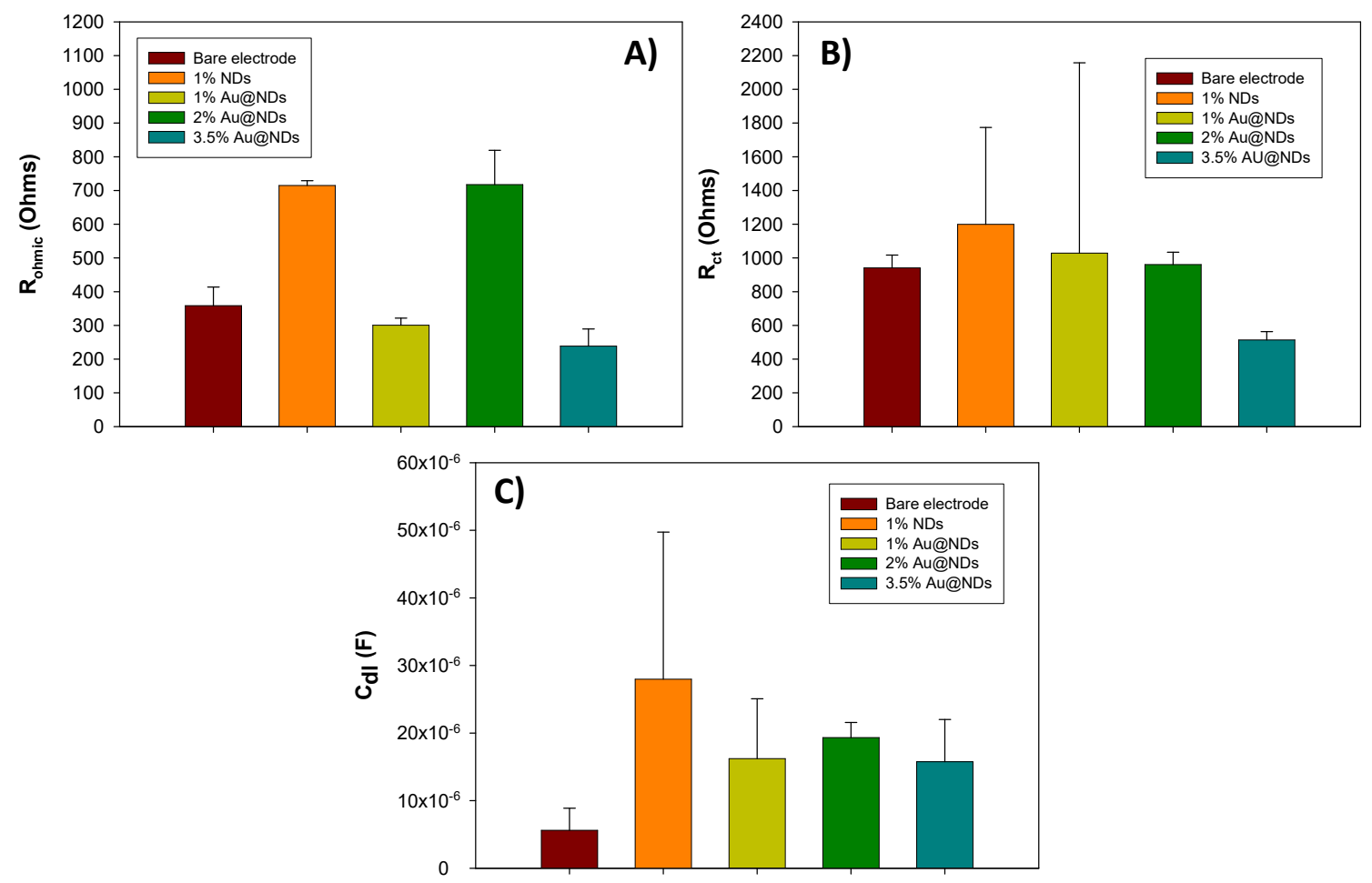

Figure 2. Graphs and values for the resistance of the solution $\left(R_{\Omega}\right)(\mathbf{A})$, electron-transfer rate $\left(R_{c t}\right)(\mathbf{B})$, and double-layer capacitance $\left(C_{d l}\right)(\mathrm{C})$ of the electrodes with $17 \%$ graphite (bare electrode), $17 \%$ graphite plus $1 \%$ raw NDs, and $17 \%$ graphite plus 1\%, 2\%, and 3.5\% Au@NDs with their corresponding standard deviation $(n=3)$. Values were extracted from the adjustment of the impedance spectrum that were obtained in a $0.1 \mathrm{KCl}$ solution with an equimolar concentration of $\left[\mathrm{Fe}(\mathrm{CN})_{6}\right]^{3-} /\left[\mathrm{Fe}(\mathrm{CN})_{6}\right]^{4-} 0.01 \mathrm{M}$. Frequencies interval: $100 \mathrm{KHz}-100 \mathrm{mHz}$.

Figure 3 compares the values for $R_{\Omega}, R_{c t}$, and $C_{d l}$ of the $17 \%$ graphite electrode, $17 \%$ graphite plus $1 \%$ raw NDs electrode, and 17\% graphite plus 1\%, 2\%, and 3\% Ag@NDs electrodes. Looking at the resistances' values, there is a decrease in the ohmic resistance and charge-transfer resistance when the amount of Ag@NDs increases. The charge-transfer resistance compared to the bare electrode is lower, so the incorporation of the Ag@NDs on the matrix produces an enhancement on the electrode sensitivity and response time. Taking into the account these values, it could be determined that the electrode with $17 \%$ graphite plus $2 \%$ Ag@NDs would be the optimal composition, but it is necessary to consider the double-layer capacitance values as well. There is an increase in this value when the amount of Ag@NDs also increases. Compared to the bare electrode $\left(5.62 \times 10^{-6} \mathrm{~F}\right)$, the electrode with $2 \% \mathrm{Ag} @ \mathrm{NDs}\left(2.16 \times 10^{-5} \mathrm{~F}\right)$ has a high value of this parameter (greater than the $1 \% \mathrm{Ag} @ \mathrm{NDs}$, $6.23 \times 10^{-6} \mathrm{~F}$ ) and it could lead to a high signal-to-noise ratio or even mask the faradic signal. According to these results, the $1 \% \mathrm{Ag} @$ NDs electrode would be the optimal since there is not much difference in the resistance values $\left(R_{\Omega}\right.$ and $\left.R_{c t}\right)$, and the double-layer capacitance value is half of the $2 \% \mathrm{Ag} @ \mathrm{NDs}$. 


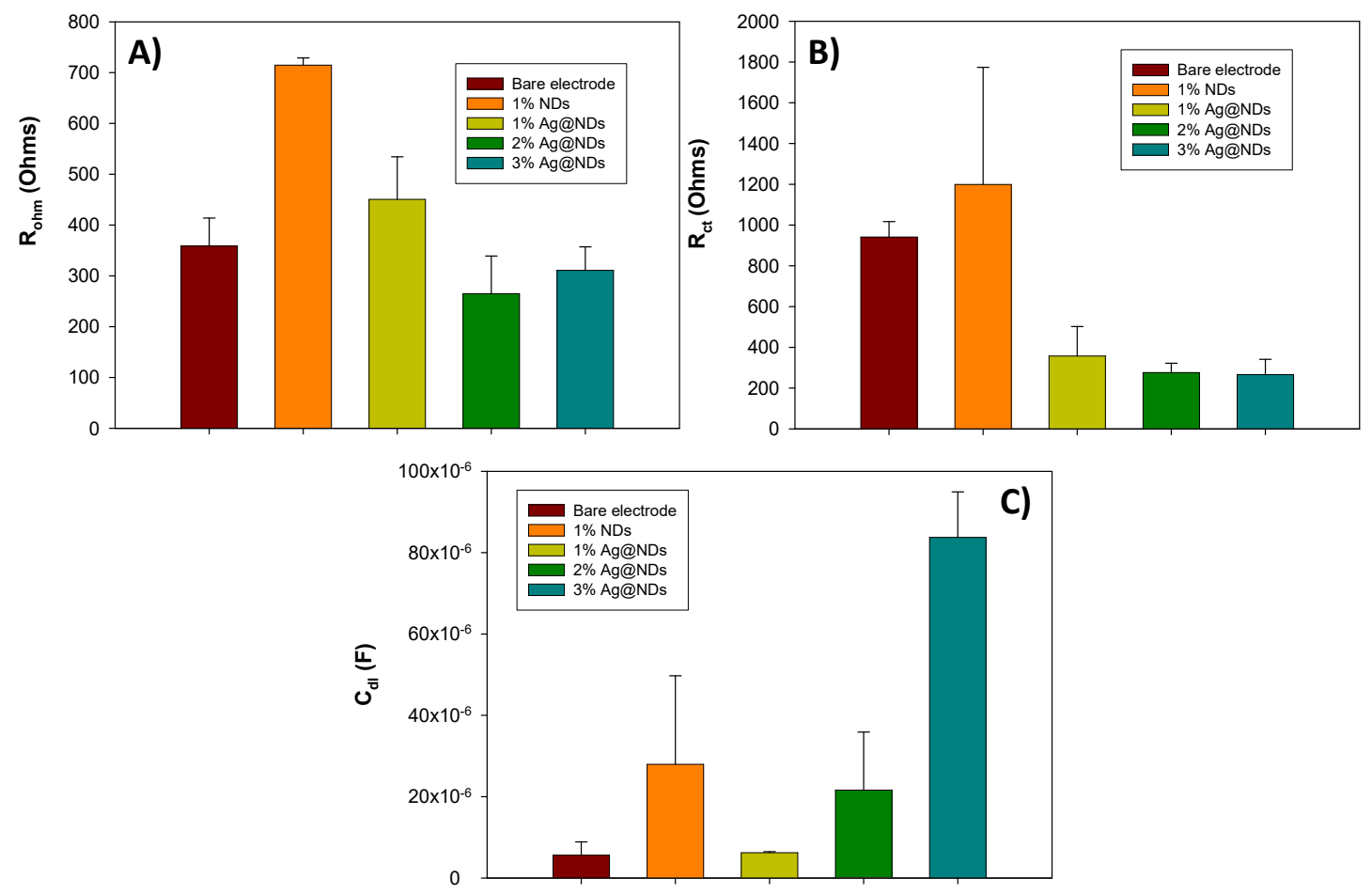

Figure 3. Graphs and values for the $R_{\Omega}(\mathbf{A}), R_{c t}(\mathbf{B})$, and $C_{d l}(\mathbf{C})$ of electrodes with $17 \%$ graphite (bare electrode), 17\% graphite plus 1\% raw NDs, and 17\% graphite plus 1\%, 2\%, and 3.5\% Ag@NDs with their corresponding standard deviation $(n=3)$. Values were extracted from the adjustment of the impedance spectrum that were obtained in a $0.1 \mathrm{M} \mathrm{KCl}$ solution with an equimolar concentration of $\left[\mathrm{Fe}(\mathrm{CN})_{6}\right]^{3-} /\left[\mathrm{Fe}(\mathrm{CN})_{6}\right]^{4-} 0.01 \mathrm{M}$. Frequencies interval: $100 \mathrm{KHz}-100 \mathrm{mHz}$.

The comparison between the optimal Au@NDs proportion (3.5\%) and the optimal Ag@NDs proportion $(1 \%)$ was made in order to evaluate the effect of both compositions of metallic load. Looking at the $R_{\Omega}$ values it can be seen that a slight difference between them, $238.64 \Omega$ for the optimal with Au@NDs and $450.57 \Omega$ for the optimal with Ag@NDs, could make the electrode with $1 \%$ of Au@NDs present better sensitivity and low response time. However, when it is compared to the $R_{c t}$ value, the Au@NDs electrode $(513.54 \Omega$ ) is higher than the resistance of the Ag@NDs electrode ( $357.85 \Omega$ ). The $C_{\mathrm{dl}}$ value of the Au@NDs $\left(1.57 \times 10^{-5} \mathrm{~F}\right)$ electrode is twice the $\mathrm{Ag} @ \mathrm{NDs}\left(6.23 \times 10^{-6} \mathrm{~F}\right)$ electrode's capacitance. For this reason and taking into the account the properties required for electroanalytical purposes, such as a rapid response time, low limit of detection, and high sensitivity, the 1\% Ag@NDs electrode is better than the $3.5 \% \mathrm{Au} @ \mathrm{NDs}$ in terms of electrochemical properties.

\subsection{Cyclic Voltammetry Measurements}

Cyclic voltammograms were taken for the different NDs containing nanocomposite electrodes and have been compared to the bare electrode based on graphite-epoxy (Figure 4).

In Figure 4A, we can see the comparison of the cyclic voltammograms regarding the electrodes containing raw NDs and Ag@NDs, and their comparison to the bare electrode. A slight difference between the voltamperograms can be seen. However, when comparing the 1\% Ag@NDs electrode to the 1\% raw NDs electrode, the oxidation-reduction peaks are closer for Ag@NDs than for raw NDs. If the oxidation peak and the reduction peak are very close to each other, it indicates a macroelectrode array behavior [23], in which the lineal diffusion is dominant. On the contrary, if both peaks are separated from one another, it indicates a microelectrode array behavior $[23,24]$ and the radial diffusion will control the mass transport. When developing this kind of transducer, the microelectrode behavior is the interesting one since this will provide an optimal particle distribution that at the same time will assure lower noise levels because there is a less active area. In this case, the variations from one peak 
to the other are not very significant (small variations) so it can be concluded that all these electrodes have a microelectrode array behavior.
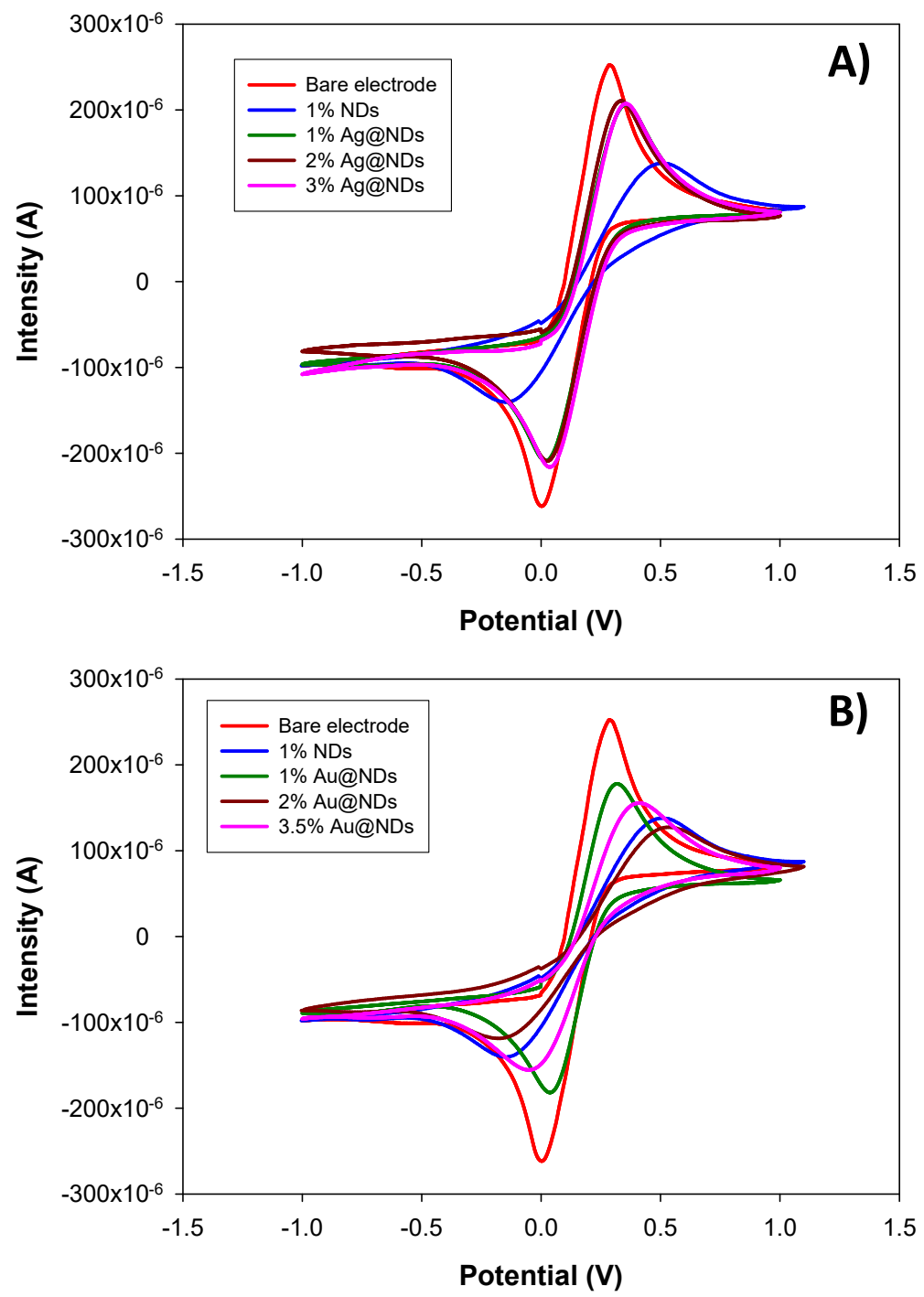

Figure 4. Superposition of the cyclic voltammograms (CV) obtained for the electrodes with $17 \%$ graphite, 17\% graphite plus 1\%, 2\%, and 3\% Ag@NDs (A). Superposition of the CV obtained for electrodes with $17 \%$ graphite, $17 \%$ graphite plus $1 \%$ raw NDs, and $17 \%$ graphite plus $1 \%, 2 \%$, and $3.5 \%$ $\mathrm{Au} @ \mathrm{NDs}(\mathbf{B})$. Scan rate: $10 \mathrm{mV} \cdot \mathrm{s}^{-1}$.

Figure $4 \mathrm{~B}$ presents the comparison between the bare electrode and the electrodes modified with different proportions of Au@NDs and raw NDs, obtaining the same trend of the previous case. However, an increase in the amount of Au@NDs is causing an offset between the cathode and anodic peak. The voltamperograms present a behavior more similar to the one with the raw NDs than to the bare electrode. In general, these results indicate that the electrodes with different proportions of the developed Au@NDs and Ag@NDs present good reproducibility and similar electrochemical behavior.

From the cyclic voltamperograms, different parameters as the peak intensity, the separation potential (between the oxidation and reduction peak), and the active area of the electrode can be extracted. The active area of the electrode can be estimated from the values of the peak intensities obtained by Randles-Sevčik (Equation (5)) [22]: Where $\alpha$ corresponds to the transference coefficient (0.5), $D_{\text {red }}$ is the diffusion coefficient of the reduced species $\left(6.32 \times 10^{-6} \mathrm{~cm}^{2} \cdot \mathrm{s}^{-1}\right), v$ is the scanning 
speed $\left(0.01 \mathrm{~V} \cdot \mathrm{s}^{-1}\right), \mathrm{A}$ is the electroactive area, and $C^{*}$ red is the bulk concentration of the electroactive species $(0.01 \mathrm{M})$.

$$
I_{p}=3.01 \times 10^{5} n^{3 / 2}\left(\alpha D_{\text {red }} v\right)^{1 / 2} \mathrm{~A} \mathrm{C}^{*} \text { red }
$$

The values for the electroactive area of each electrode are listed in Table 2. The bare electrode containing only graphite presents an electroactive area of $0.43 \pm 0.09 \mathrm{~cm}^{2}$ but when it is modified with $1 \%$ of NDs there is a decrease on the electroactive area (down to $0.27 \pm 0.03 \mathrm{~cm}^{2}$ ) as the NDs are separating the conductive particles present on the matrix. This separation of the conductive particles is compensated by the previous functionalization of the NDs with Ag and Au nanoparticles. It is verified that when increasing the percentage of NP@NDs the electroactive area increases, in addition the Ag functionalization supposes a greater increase. This fact can be explained by the intrinsic differences in the process of functionalization by galvanic replacement [18,20]. During the process of functionalization of NDs with $\mathrm{Au}$, three $\mathrm{Ag}$ atoms are replaced galvanically by one Au atom. Consequently, the resulting amount of Au in Au@NDs is lower than the amount of Ag in Ag@NDs and the electroactive area is lower. When the matrix is modified with different proportions of Ag@NDs, there is a clear upward trend: The more Ag@NDs percentage the higher the electroactive area the electrode presents. This is due to the incorporation of conducting particles that are introduced into the matrix, although this fact modifies the spatial separation and inner distribution of the conductive particles.

Table 2. Values of the electroactive area for the different developed electrodes. The experimental error is calculated as the standard deviation for $n=3$ measurements.

\begin{tabular}{cccc}
\hline Electrode & Electroactive Area $\left.\mathbf{( c m}^{\mathbf{2}}\right)$ & Electrode & Electroactive Area $\left.\mathbf{( c m}^{\mathbf{2}}\right)$ \\
\hline $17 \%$ graphite & $0.43 \pm 0.09$ & $\begin{array}{c}17 \% \text { graphite } \\
+1 \% \text { NDs }\end{array}$ & $0.27 \pm 0.03$ \\
\hline $\begin{array}{r}17 \% \text { graphite } \\
+1 \% \text { Ag@NDs }\end{array}$ & $0.39 \pm 0.01$ & $\begin{array}{c}17 \% \text { graphite } \\
+1 \% \text { Au@NDs }\end{array}$ & $0.30 \pm 0.06$ \\
\hline $\begin{array}{l}17 \% \text { graphite } \\
+2 \% \text { Ag@NDs }\end{array}$ & $0.40 \pm 0.01$ & $\begin{array}{c}17 \% \text { graphite } \\
+2 \% \text { Au@NDs }\end{array}$ & $0.23 \pm 0.01$ \\
\hline $\begin{array}{l}17 \% \text { graphite } \\
+3 \% \text { Ag@NDs }\end{array}$ & $0.42 \pm 0.03$ & $\begin{array}{l}17 \% \text { graphite } \\
+3.5 \% \text { Au@NDs }\end{array}$ & $0.29 \pm 0.01$ \\
\hline
\end{tabular}

For electrodes with $\mathrm{Au} @ \mathrm{NDs}$, it seems that higher percentages of Au@NDs are needed to increase the electroactive area with respect to the $1 \%$ raw NDs electrode. To compensate this problem, a higher percentage of the conducting material (Au NPs) may be necessary, to obtain an electroactive area that is similar to the case of $1 \%$ raw NDs.

Taking into the account the results obtained by EIS and CV techniques, it is suggested that the nanocomposite modified with 1\% of Ag@NDs seems to be the optimal proportion in terms of the electrochemical response features.

\subsection{Electroanalytical Evaluation}

Once the best composition regarding the electrochemical properties for the electrodes based on graphite-epoxy and Ag@NDs was determined (17\% graphite + 1\% Ag@NDs), the electroanalytical properties of the electrode were evaluated and compared to the ones for the bare electrode. Firstly, the characterization was done by the determination of hydrogen peroxide as a model analyte.

\subsubsection{Evaluation of the Working Potential}

To study the catalytic effect of introducing small amounts of Ag@NDs in the electrode's matrix, firstly the polarization potential for hydrogen peroxide has to be determined. To do so, a study using the linear sweep voltammetry (LSV) technique was carried out at different concentrations of analyte 
and in the absence of analyte for two of the electrodes: The one containing 1\% Ag@NDs, which was found to present optimal electrochemical properties, and the one without any modifier (bare electrode).

Figure S3 (see Supplementary Materials) shows both lineal voltamperograms for the electrode containing only the graphite-epoxy (17\% graphite) and the Ag@NDs containing electrode (17\% graphite $+1 \%$ Ag@NDs). The oxidation of the hydrogen peroxide using the bare electrode is obtained at $900 \mathrm{mV}$. However, for the electrode modified with Ag@NDs the potential is reduced to $750 \mathrm{mV}$. Therefore, the introduction of the Ag@NDs in the nanocomposite matrix enhances the electrochemical detection of this analyte reducing the working potential by $17 \%$. This fact confers selectivity to the sensor because it decreases the number of species that can be oxidized.

\subsubsection{Electroanalytical Parameters of Response}

The electroanalytical parameters of response such as sensitivity and limit of detection have been compared for both sensors (bare and 1\% Ag@NDs electrode). Measurements were carried out using the hydrodynamic amperometry technique at $750 \mathrm{mV}$ as the working potential. The LOD measurements were performed by triplicate and experimental errors are expressed as the standard deviation. By adding 1\% Ag@NDs to the composite transducers, no significant differences have been observed regarding the sensitivity, $0.01 \mu \mathrm{A} \cdot \mathrm{L} \cdot \mathrm{mg}^{-1}$ for bare electrode and $0.09 \mu \mathrm{A} \cdot \mathrm{L} \cdot \mathrm{mg}^{-1}$ for 1\% Ag@NDs. A similar trend is observed regarding the limit of detection for hydrogen peroxide, $0.068 \pm 0.001$ and $0.095 \pm 0.003 \mathrm{mg} \cdot \mathrm{L}^{-1}$ for bare electrode and 1\% Ag@NDs, respectively. However, what this modified electrode offers is the possibility of working at lower potentials and thus reducing the interferences due to the presence of other analytes that can be oxidized at the same potential, as mentioned previously. The reduction of the working potential is a considerable improvement since one of the main problems of amperometric (bio)sensors is the fact that high working potentials are needed unless a redox mediator is used. The introduction of this kind of nanoparticle could be an alternative to the redox mediator. In addition, this lessening of the polarization potential reduces the possible interferences since at a lower potential less species will be oxidized.

\subsection{Evaluation of (Bio)sensing Approach: Analytical Response to Glucose}

Optimized nanocomposites electrodes with 1\% Ag@NDs, have been modified with glucose oxidase as an enzyme model in order to evaluate the analytical response to glucose. Firstly, the working potential for glucose detection after the incorporation of the enzyme by LSV has been evaluated (see Figure S4, Supplementary Materials). Figure S5 (Supplementary Materials) summarizes the analytical procedure and the reaction that takes place on the electrode's surface. The complete reaction consists of two separate reactions: The first one is the biocatalyst reaction between the glucose oxidase and the glucose by hydrogen peroxidase production, and the second is the electroanalytical reaction when the product is oxidized on the surface electrode. This study was carried out in the presence of glucose after the 1\% Ag@NDs electrode was incubated with glucose oxidase for $30 \mathrm{~min}$. The results showed that the oxidation potential is $820 \mathrm{mV}$. Compared to the working potential for enzymatic (bio)sensors for glucose determination on the literature, the potential is improved by the incorporation of the nanomaterials from 1050 [16] to $820 \mathrm{mV}$, so it was reduced significantly. Consequently, the selectivity when the enzyme is placed on the surface of the Ag@NDs-tuned electrode, with respect to matrix modification was increased.

In order to optimize the experimental conditions, an evaluation of the influence of the contact time in the electroanalytical parameters of response has been done. The times studied were $30 \mathrm{~min}$, $1 \mathrm{~h}$ and $24 \mathrm{~h}$. Lower incubation times were evaluated but there was no response to glucose. Figure 5 shows the calibration curve (A) and the linear range (B) for the electrode with $17 \%$ graphite plus $1 \%$ Ag@NDs for an incubation with GOD of $30 \mathrm{~min}$ and $1 \mathrm{~h}$. When the electrode surface was incubated for $24 \mathrm{~h}$, the electrode's response was saturated at lower concentrations $(0.7 \mathrm{mM})$, so this experiment is not included in the Figure. Therefore, at this point it can be concluded that $24 \mathrm{~h}$ of contact time are not necessary in order to obtain good sensitivity, low detection limit, and a wide linear range. 

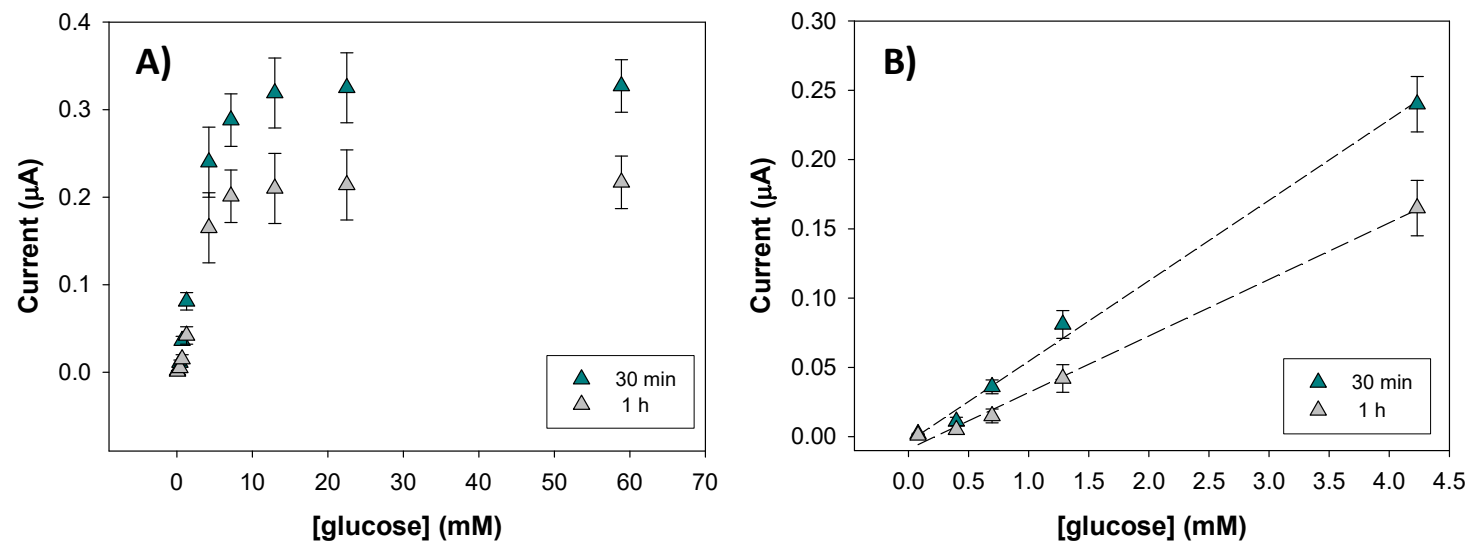

Figure 5. Response of the 17\% graphite + 1\% Ag@NDs electrodes after being mixed with glucose oxidase in the thermomixer for $30 \mathrm{~min}$ and $1 \mathrm{~h}$ for glucose concentration between 0-60 $\mathrm{mM}(\mathrm{A})$. Linear concentration range for $t=30 \mathrm{~min}$ and $t=1 \mathrm{~h}(\mathbf{B})$. The experimental error was calculated as the standard deviation for $n=3$ measurements.

Table 3 details the analytical parameters such as detection limit and sensitivity. It can be determined that there is no significant difference observed on sensitivity and detection limit when the time contact increases from $30 \mathrm{~min}$ to $1 \mathrm{~h}$. However, the increase in the contact time produces a higher enzyme saturation at a lower glucose concentration (Figure 5A). According to these results, and in order to reduce the contact time, 30 min were considered as the optimal contact time.

Table 3. Calibration parameters obtained with the hydrodynamic amperometry technique for the electrode 1\% graphite + 1\% Ag@NDs modified with GOD by the direct contact method at different contact times, using glucose as the analyte. The experimental error was calculated as the standard deviation for $n=3$ measurements.

\begin{tabular}{cccc}
\hline Time & Sensitivity $\left(\boldsymbol{\mu A} \cdot \mathbf{m M}^{-\mathbf{1}}\right)$ & LOD $(\mathbf{m M})$ & Saturation Concentration $(\mathbf{m M})$ \\
\hline $30 \mathrm{~min}$ & $0.058 \pm 0.002$ & $0.08 \pm 0.01$ & 12.98 \\
$1 \mathrm{~h}$ & $0.041 \pm 0.003$ & $0.08 \pm 0.01$ & 7.16 \\
$24 \mathrm{~h}$ & - & - & 0.70 \\
\hline
\end{tabular}

Compared to other (bio)sensors based on nanomaterials [25], the LOD achieved are similar ( 0.09 vs. $0.08 \mathrm{mM}$ in this work), so this highlights the potential of the developed (bio)sensor platform. The limit of detection achieved with a biocomposite electrode based on graphite-epoxy (17:82 $(w / w))$ and modified with $1 \%$ of GOD in the matrix [16] compared with the presented platform are similar: 0.04 and $0.08 \mathrm{mM}$, respectively. However, the sensitivity decreases one order of magnitude, from 0.214 to $0.058 \mu \mathrm{A} / \mathrm{mM}$. It is important to highlight that the (bio)nanocomposite electrodes presented in this work containing a lower enzyme amount achieve an equivalent limit of detection towards glucose.

In order to evaluate the specific adsorption of glucose oxidase on the electrode surface, the bare electrode was incubated for the optimal time of $30 \mathrm{~min}$ with the enzyme solution. No changes on the current were observed after the glucose additions. Therefore, this means that the specific adsorption of the glucose oxidase on the surface of the electrode only takes place when there are Ag@NDs that are incorporated to the electrode matrix, as expected. The stability of the developed device was evaluated by the hydrodynamic amperometry. The short-term stability of the platform was investigated by repeating the determination of 0.4 and $4.2 \mathrm{mM}$ glucose aliquots at the same sensor. Nine measurements for each concentration achieved a good repeatability with the relative standard deviation (RSD) of $0.03 \%$ and $0.04 \%$, respectively. Afterwards, multiple calibration experiments were performed with the same nanocomposite sensor within a $24 \mathrm{~h}$ period in order to make an estimation of the loss of sensitivity to long-time. During a working day, the sensor was exposed to air without renewing their 
surface and no more functionalization process was required. Six calibrates were made and a sensitivity of $0.06 \pm 0.01 \mu \mathrm{A} \cdot \mathrm{mM}^{-1}(n=6)$ and $\mathrm{RSD}$ of $3.74 \%$ was obtained. The low RSD value can be mainly attributed to the chemical stability of the enzyme over surface of the transducer.

\section{Conclusions}

In this work, nanocomposite sensors containing different proportions of Ag@NDs and Au@NDs were constructed and characterized by means of electrochemical impedance spectroscopy and cyclic voltammetry in order to optimize their analytical response in terms of high signal-to-noise ratio, rapid response, and low limit detection. In this sense, EIS and CV allowed us to optimize the nanocomposite composition regarding ohmic resistance (related to percolation theory), charge-transfer resistance (associated to heterogeneous electron-transfer, which depends on the electrochemical active surface), and double-layer capacitance (correlated to the background current and consequently to the signal/noise ratio). In the present work, the optimal composition for Au@NDs present on the nanocomposite matrix is 3.5\% and the one for Ag@NDs is 1\%. When comparing both optimal modifier compositions, 1\% Ag@NDs on the electrode turned out to be the best one since despite presenting a higher ohmic resistance, it offered a lower charge-transfer resistance and double-layer capacitance.

The electroanalytical experiments showed that the incorporation of 1\% Ag@NDs on the graphite/epoxy matrix allow reducing the working potential for the hydrogen peroxide from $900 \mathrm{mV}$ using the bare electrode to $750 \mathrm{mV}$, which is an important feature in amperometric measurements in order to minimize the interferences.

Good results were obtained in the immobilization of an enzyme on the electrode's surface by direct contact. The electroanalytical evaluation of glucose using the nanocomposite sensor tuned with GOD allowed reducing the working potential from 1050 to $820 \mathrm{mV}$ achieving a good detection limit and sensitivity.

In conclusion, we developed an easy to develop, cheap, and robust electrochemical sensor based on nanocomposite material formed of 17\% graphite and 1\% Ag@NDs. This kind of electrode could become a suitable platform in terms of sensing, as long as the enzymatic reaction taking place on its surfaces produces hydrogen peroxide (any type of oxidase) that will be oxidized directly on the electrode's surface. Moreover, further research could be addressed to enlarge this line of interest in terms of a different functionalization of the electrodes' surface to widen the detection possibilities and applications.

Supplementary Materials: The following are available online at http://www.mdpi.com/2079-4991/10/6/1179/s1; Figure S1: SEM images of the graphite/epoxy electrodes; Figure S2: Nyquist plots and scheme of the equivalent circuit; Figure S3: Linear sweep voltamperograms using hydrogen peroxide as analyte; Figure S4: Linear sweep voltamperogram obtained for the 1\% Ag@NDs electrode modified with GOD; Figure S5: Scheme of the analytical procedure.

Author Contributions: Funding acquisition, M.B.; investigation, R.M., G.S., J.Z., C.P. and J.B.-A.; project administration, M.B.; supervision, R.M., C.P., J.B.-A. and M.B.; writing-original draft, R.M., G.S., J.Z., C.P., J.B.-A. and M.B.; writing-review and editing, R.M., C.P., J.B.-A. and M.B. All authors have read and agreed to the published version of the manuscript.

Funding: This research was funded by the Spanish Ministerio de Economía y Competitividad y Fondo Europeo de Desarrollo Regional (MINECO/FEDER, UE), grant number RTI2018-099362-B-C21.

Conflicts of Interest: The authors declare no conflict of interest.

\section{References}

1. Baeza, M.; Olivé-Monllau, R.; Esplandiu, M.J. Advances on rigid conducting composites for electroanalytical applications. In Resin Composites: Properties, Production and Applications; Deborah, B.S., Ed.; Nova Science Publishers, Inc.: Hauppauge, NY, USA, 2011; pp. 153-211, ISBN 9781612091297.

2. Esplandiu, M.J.; Baeza, M.; Olive-Monllau, R.; Cespedes, F.; Bartroli, J. Development of Tunable Nanocomposites Made from Carbon Nanotubes for Electrochemical Applications. In Advances in Composite Materials for Medicine and Nanotechnology; Attaf, B., Ed.; InTech: London, UK, 2011; p. 289, ISBN 978-953-307-235-7. 
3. Murray, R.W. Nanoelectrochemistry: Metal nanoparticles, nanoelectrodes, and nanopores. Chem. Rev. 2008, 108, 2688-2720. [CrossRef] [PubMed]

4. Hugelmann, M.; Hugelmann, P.; Lorenz, W.J.; Schindler, W. Nanoelectrochemistry and nanophysics at electrochemical interfaces. Surf. Sci. 2005, 597, 156-172. [CrossRef]

5. Mochalin, V.N.; Shenderova, O.; Ho, D.; Gogotsi, Y. The properties and applications of nanodiamonds. Nat. Nanotechnol. 2011, 7, 11-23. [CrossRef] [PubMed]

6. Wang, J. Nanomaterial-based electrochemical biosensors. Analyst 2005, 130, 421. [CrossRef]

7. Merko, A.; Ambrosi, A.; De, A.; Guix, M.; Maltez, M.; Marin, S.; Merkoçi, A.; Ambrosi, A.; De la Escosura-Muñiz, A.; Guix, M.; et al. Nanomaterials for Electroanalysis. Encycl. Anal. Chem. 2010. [CrossRef]

8. Briones, M.; Petit-Domínguez, M.D.; Parra-Alfambra, A.M.; Vázquez, L.; Pariente, F.; Lorenzo, E.; Casero, E. Electrocatalytic processes promoted by diamond nanoparticles in enzymatic biosensing devices. Bioelectrochemistry 2016, 111, 93-99. [CrossRef]

9. Baccarin, M.; Rowley-Neale, S.J.; Cavalheiro, É.T.G.; Smith, G.C.; Banks, C.E. Nanodiamond based surface modified screen-printed electrodes for the simultaneous voltammetric determination of dopamine and uric acid. Microchim. Acta 2019. [CrossRef]

10. Krolow, M.; Hartwig, C.; Link, G.; Raubach, C.; Pereira, J.; Picoloto, R.; Gonçalves, M.; Carreño, N.; Mesko, M. Synthesis and Characterization of Carbon Nanocomposites. In Carbon Nanostructures; Springer: Berlin/Heidelberg, Germany, 2013; pp. 33-47.

11. Muñoz, J.; Montes, R.; Baeza, M. Trends in electrochemical impedance spectroscopy involving nanocomposite transducers: Characterization, architecture surface and bio-sensing. TrAC-Trends Anal. Chem. 2017, 97. [CrossRef]

12. Bastos-Arrieta, J.; Montes, R.; Ocaña, C.; Espinoza, M.; Muñoz, M.; Baeza, M. In situ characterization of size, spatial distribution, chemical composition, and electroanalytical response of hybrid nanocomposite materials. In In-Situ Characterization Techniques for Nanomaterials; Springer: Berlin/Heidelberg, Germany, 2018; ISBN 9783662563229.

13. Montes, R.; Bartrolí, J.; Céspedes, F.; Baeza, M. Towards to the improvement of the analytical response in voltammetric sensors based on rigid composites. J. Electroanal. Chem. 2014, 733, 69-76. [CrossRef]

14. Esplandiu, M.J.; Pacios, M.; Cyganek, L.; Bartroli, J.; del Valle, M. Enhancing the electrochemical response of myoglobin with carbon nanotube electrodes. Nanotechnology 2009, 20, 355502. [CrossRef]

15. Costentin, C.; Drouet, S.; Robert, M.; Savéant, J.-M. Turnover numbers, turnover frequencies, and overpotential in molecular catalysis of electrochemical reactions. Cyclic voltammetry and preparative-scale electrolysis. J. Am. Chem. Soc. 2012, 134, 11235-11242. [CrossRef] [PubMed]

16. Montes, R.; Bartrolí, J.; Baeza, M.; Céspedes, F. Improvement of the detection limit for biosensors: Advances on the optimization of biocomposite composition. Microchem. J. 2015, 119, 66-74. [CrossRef]

17. Montes, R.; Céspedes, F.; Baeza, M. Highly sensitive electrochemical immunosensor for IgG detection based on optimized rigid biocomposites. Biosens. Bioelectron. 2016, 78, 505-512. [CrossRef] [PubMed]

18. Bastos-Arrieta, J.; Muñoz, J.; Vigués, N.; Muraviev, D.N.; Céspedes, F.; Mas, J.; Baeza, M.; Muñoz, M. Intermatrix synthesis of $\mathrm{Ag}, \mathrm{AgAu}$ and $\mathrm{Au}$ nanoparticles by the galvanic replacement strategy for bactericidal and electrocatalytically active nanocomposites. New J. Chem. 2016, 40, 10344-10352. [CrossRef]

19. Bastos-Arrieta, J.; Muñoz, J.; Stenbock-Fermor, A.; Muñoz, M.; Muraviev, D.N.; Céspedes, F.; Tsarkova, L.A.; Baeza, M. Intermatrix Synthesis as a rapid, inexpensive and reproducible methodology for the in situ functionalization of nanostructured surfaces with quantum dots. Appl. Surf. Sci. 2016, 368, 417-426. [CrossRef]

20. Muñoz, J.; Bastos-Arrieta, J.; Muñoz, M.; Muraviev, D.; Céspedes, F.; Baeza, M. Simple green routes for the customized preparation of sensitive carbon nanotubes/epoxy nanocomposite electrodes with functional metal nanoparticles. RSC Adv. 2014, 4, 44517-44524. [CrossRef]

21. Kodera, F.; Umeda, M.; Yamada, A. Determination of free chlorine based on anodic voltammetry using platinum, gold, and glassy carbon electrodes. Anal. Chim. Acta 2005. [CrossRef]

22. Pacios, M.; del Valle, M.; Bartroli, J.; Esplandiu, M.J. Electrochemical behavior of rigid carbon nanotube composite electrodes. J. Electroanal. Chem. 2008, 619-620, 117-124. [CrossRef]

23. Nirmaier, H.-P.; Henze, G. Characteristic behavior of macro-, semimicro- and microelectrodes in voltammetric and chronoamperometric measurements. Electroanalysis 1997, 9, 619-624. [CrossRef] 
24. Gabrielli, C.; Keddam, M.; Portail, N.; Rousseau, P.; Takenouti, H.; Vivier, V. Electrochemical Impedance Spectroscopy Investigations of a Microelectrode Behavior in a Thin-Layer Cell: Experimental and Theoretical Studies. J. Phys. Chem. B 2006, 110, 20478-20485. [CrossRef]

25. Pérez, B.; Pumera, M.; Del Valle, M.; Merkoçi, A.; Alegret, S. Glucose biosensor based on carbon nanotube epoxy composites. J. Nanosci. Nanotechnol. 2005. [CrossRef] [PubMed]

(C) 2020 by the authors. Licensee MDPI, Basel, Switzerland. This article is an open access article distributed under the terms and conditions of the Creative Commons Attribution (CC BY) license (http://creativecommons.org/licenses/by/4.0/). 